\title{
Desenvolvimento inicial de larvas de Rhaphiodon vulpinus Agassiz (Characiformes, Cynodontidae)
}

\author{
Wilson Treger Zydowicz de Sousa ${ }^{1}$ \\ William Severi $^{1}$
}

\begin{abstract}
Initial development of Rhaphiodon vulpinus Agassiz larvae (Characiformes, Cynodontidae). The cynodontid fish Rhaphiodon vulpinus Agassiz, 1829, known as "peixe-cachorro", is the only species of the family occurring in the Pantanal of Mato Grosso. The initial development of its larvae was characterized, based on material collected with a $500 \mu \mathrm{m}$ mesh size plankton-net, in several biotopes of the Pantanal of Barão de Melgaço, State of Mato Grosso, Central Brazil. The analysis was carried out with 72 larvae with standard length (SL) between 4.7 and $13.8 \mathrm{~mm}$. The morphometric measurements varied positively with standard length, and body proportions ranged $8.1-17.8 \%$ for head length (HL), 6.2-10.1\% for body height, and 63.6$73.5 \%, 16.8-21.6 \%$, and $61.8-78.2 \% \mathrm{SL}$, respectively for pre-dorsal, pre-pectoral and pre-anal distances. The eye diameter varied positively with HL and its proportion varied between $9.1-22.5 \% \mathrm{HL}$. The body relations did not show great variation along the larval development. The morphological characterization of larvae from 4.7 to $5.8 \mathrm{~mm} \mathrm{SL}$, in the pre-flexion phase, showed the presence of an embryonic finfold covering the unrayed fins, exposed branchial arches, absence of exogenous digestive content, unpigmented eyes and round shaped head. Larvae with SL between 8.1 and $13.8 \mathrm{~mm} \mathrm{SL}$ were in the flexion phase, presenting a pointed head, mouth filled with external and internal rows of numerous teeth, presence of exogenous food, pigmented eyes, branchial filaments still exposed, evident swimming-bladder, and formation of the first caudal, dorsal and anal fins' rays. Only individuals in the pre-flexion and flexion phases were analyzed, and no completely developed fin was evidenced. A maximum number of 69 myomeres was counted and no evident pigmentation over the body observed. Comparing $R$. vulpinus larvae to those of other Characiformes, it was observed that this species and Apareiodon affinis Steindachner, 1879 present smaller body proportions, and variation range of body height, while $R$. vulpinus is characterized by a shorter eye diameter. The myomere number is a relevant feature for the identification of $R$. vulpinus larvae, since it is the Characiform that possesses the highest myomere number, among those species of this family studied so far.
\end{abstract}

KEY WORDS. Cynodontidae, Rhaphiodon vulpinus, larval development, ontogeny, morphometrics

O estudo da ecologia das fases iniciais do ciclo vital e da ontogenia em peixes está historicamente associado à biologia pesqueira e ao recrutamento de espécies marinhas, principalmente daquelas de importância comercial, sendo considerável o volume de informações sobre espécies de água salgada (BLAXTER 1984). Uma sinopse histórica e levantamento bibliográfico podem ser encontrados em MOSER et al. (1984), LASKER (1987) e ABLE \& FAHAY (1998).

1) Laboratório de Ictiologia, Departamento de Pesca, Universidade Federal Rural de Pernambuco. Rua Dom Manuel de Medeiros, Dois Irmãos, 52171-900 Recife, Pernambuco, Brasil. E-mail: wseveri@truenet.com.br

Revta bras. Zool. 19 (1): $85-94,2002$ 
O conhecimento sobre o desenvolvimento larval de peixes de água doce, sobretudo para espécies neotropicais, é entretanto, limitado. A despeito de algumas descrições de larvas de Characiformes, Perciformes e Siluriformes realizadas nos últimos 20 anos (e.g. OlDANI 1977, 1979a,b, 1983a,b; ARAÚJO-LIMA 1985, 1991; NAKATANI et al. 1997, 1998), o conhecimento sobre a ecologia e a ontogenia de espécies destas ordens, de relevante importância pesqueira para a região, pouco mudou desde a revisão de MOSER et al. (1984).

Rhaphiodon vulpinus Agassiz, 1829, vulgarmente conhecido como peixecachorro, dourado-cachorro ou dourada-cadela, é um Characiformes amplamente distribuído nas bacias dos rios Orinoco, Amazonas, Araguaia, Tocantins, Uruguai e Paraná-Paraguai (Fowlen 1950; GOULDING 1980; GODOY 1986; BRITSKI et al. 1999; TOLEDO-PIZA 2000), sendo a única espécie dentre os três gêneros da subfamília Cynodontinae - Hydrolicus Müller \& Troschel, 1844, Cynodon Agassiz, 1829 e Rhaphiodon Agassiz, 1829 - (LUCENA \& MENEZES 1998), com ocorrência no Pantanal Mato-grossense. É um peixe facilmente reconhecível, por apresentar a peculiaridade de mover sua cabeça para cima, num ângulo de $45^{\circ}$, o que confere-lhe um perfil característico (LESIUK \& LINDSEY 1978).

O presente trabalho tem como objetivo contribuir para a ampliação do conhecimento sobre a ontogenia de $R$. vulpinus, através da caracterização de seu desenvolvimento inicial com a descrição da morfologia externa e morfometria de larvas da espécie.

\section{MATERIAL E MÉTODOS}

Amostras mensais de ictioplâncton foram obtidas através de arrastos diurnos e horizontais de superfície, empregando-se rede de plâncton cônico-cilíndrica com malha de $500 \mu \mathrm{m}$, no período de outubro/94 a setembro/95, efetuadas na porção

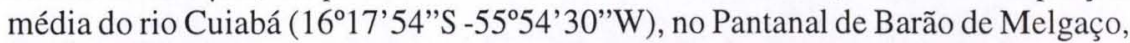
Estado de Mato Grosso. O material foi fixado in situ com formol 5\% neutralizado e triado sob estereomicroscópio.

A separação das larvas de $R$. vulpinus de outros Characiformes baseou-se em características morfológicas e merísticas, como corpo alongado e comprimido, fenda bucal inclinada, maxilas com dentes caninos anteriores, nadadeira dorsal localizada sobre a anal e número de miômeros variando de 65 a 68 .

As medidas e ilustrações das larvas foram obtidas com auxílio de estereomicroscópio, equipado com ocular micrométrica e câmara clara. Alguns exemplares foram corados com azul de alcian e alizarina, segundo o método de DiNGERKUS \& UHLER (1977), para melhor evidenciar as estruturas ósseas.

Foram analisadas 72 larvas de $R$. vulpinus, com comprimento padrão $(\mathrm{CP})$ variando entre 4,7 e 13,8 mm. Não foram obtidos indivíduos com CP entre 5,8 e 8,1 $\mathrm{mm}$. A caracterização de seu desenvolvimento inicial foi realizada com base numa série ontogênica (KENDALL et al. 1984), separando-se os indivíduos conforme o grau de flexão da notocorda, nas fases de pré-flexão e flexão. Não foram analisados indivíduos na fase de pós-flexão. 
Os indivíduos foram caracterizados morfometricamente, de acordo com as seguintes medidas: comprimento padrão $(\mathrm{CP})$ - focinho à extremidade da notocorda; altura do corpo (AC) - distância vertical entre as margens dorsal e ventral do corpo, anteriormente à nadadeira peitoral; comprimento da cabeça $(\mathrm{CC})$ - focinho à margem posterior do opérculo; distância pré-dorsal (DPD) - focinho ao início do finfold ou primeiro raio da nadadeira dorsal; distância pré-peitoral (DPP) - focinho à inserção do finfold da nadadeira peitoral; distância pré-anal (DPA) - focinho à margem posterior do ânus; e diâmetro do olho (DO) - distância longitudinal entre as bordas anterior e posterior do olho. As medidas AC, CC, DPD, DPP e DPA foram relacionadas com $\mathrm{CP}$ e DO com $\mathrm{CC}$, através de regressão linear.

A variação das relações corporais ao longo do desenvolvimento também foi analisada em função de $\mathrm{CP}$, exceto para $\mathrm{DO}$, expresso em relação a CC.

\section{RESULTADOS}

\section{Caracterização morfológica}

Larvas de 4,7 a 5,8 mm CP apresentaram-se na fase de pré-flexão, com notocorda retilínea. Nesta fase, uma membrana embrionária (finfold) envolve toda a região dorsal e ventral, sendo interrompida apenas pelo ânus, sem nítida diferenciação entre as nadadeiras dorsal, caudal e anal, e sem a presença de raios. Evidencia-se a presença dos arcos branquiais, sendo que o opérculo, ainda em formação, não recobre totalmente a cavidade branquial. Não há evidência de conteúdo digestivo exógeno, mas a boca encontra-se aberta, sem a presença de dentes. Os olhos não apresentam pigmentação, o que tornou difícil sua medição. Em larvas de 4,7 mm CP (Fig. 1A), a cabeça apresentava forma arredondada, tornando-se ligeiramente pontiaguda em larvas de 5,5 mm CP (Fig. 1B). Foram evidenciados 50 miômeros em larvas de $5,3 \mathrm{~mm} \mathrm{CP}$, número máximo visualizado em indivíduos de até $5,8 \mathrm{~mm} \mathrm{CP}$.

Larvas de 8,1 a 13,8 mm CP encontravam-se na fase de flexão. A cabeça, nesta fase, apresenta-se ainda mais pontiaguda, com o focinho protuberante. A boca é provida de fileiras externa e interna de numerosos dentes cônicos e os olhos encontram-se pigmentados. A bexiga natatória tornou-se visível desde $8,1 \mathrm{~mm} \mathrm{CP}$. Com 8,5 mm CP (Fig. 1C), seis raios na nadadeira caudal foram visualizados, dando início à sua formação. A membrana da nadadeira peitoral foi evidenciada a partir de 9,4 mm CP (Fig. 1D). Com 11,1 mm CP apareceram vestígios dos pterigióforos da nadadeira anal e com 11,4 mm CP (Fig. 1E), evidenciou-se o desenvolvimento dos primeiros raios da nadadeira dorsal. O número máximo de 69 miômeros foi evidenciado a partir de $11,0 \mathrm{~mm} \mathrm{CP}$.

Com 13,8 mm CP (Fig. 1F), a estrutura opercular, embora calcificada, ainda não recobria totalmente a cavidade branquial e os filamentos branquiais projetavam-se além da borda do opérculo.

A base estrutural óssea da nadadeira anal surje antes de qualquer estrutura óssea da nadadeira dorsal nas larvas analisadas de $R$. vulpinus, mas esta última nadadeira tem seus primeiros raios evidenciados antes do aparecimento de raios na anal. 
Não foram evidenciados pigmentos nas larvas analisadas. Estas apresentaram uma coloração variável entre bege claro, naquelas coletadas em áreas lacustres (baías), e marrom pardacento, naquelas coletadas na calha do rio Cuiabá.
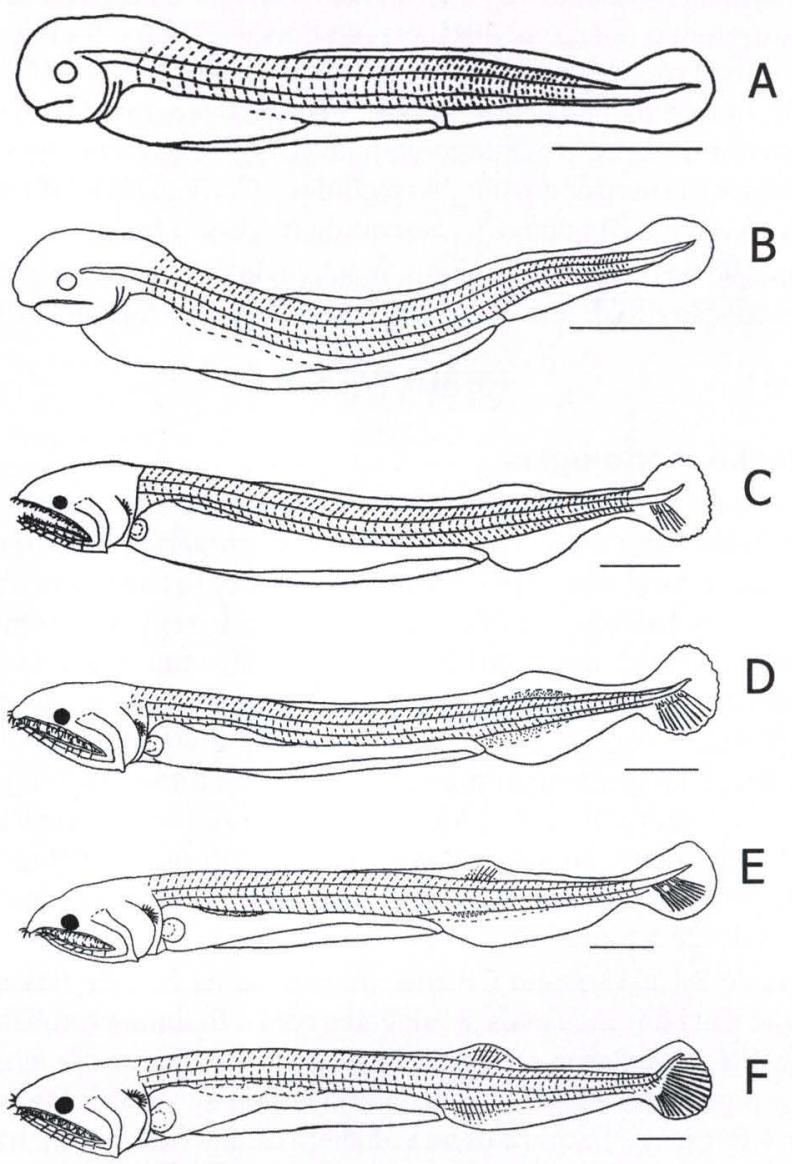

Fig. 1. Larvas de Rhaphiodon vulpinus: (A) 4,7 mm CP, fase de pré-flexão; (B) 5,5 mm CP, fase de pré-flexão; (C) $8,5 \mathrm{~mm}$ CP, fase de flexão; (D) $9,6 \mathrm{~mm}$ CP, fase de flexão; (E) 11,4 mm $\mathrm{CP}$, fase de flexão; (F) 13,8 mm CP, fase de flexão. Barra correspondente a $1 \mathrm{~mm}$.

\section{Desenvolvimento morfométrico}

A figura 2A-F apresenta, em sequência, a regressão linear entre o comprimento da cabeça, altura do corpo, a distância pré-dorsal, distância pré-peitoral e distância pré-anal em relação ao comprimento padrão, e do diâmetro do olho em relação ao comprimento da cabeça. Os coeficientes de correlação linear de Pearson (r) apresentaram valores superiores a $0,85(\mathrm{P}<0,001)$. Todas as medidas analisadas correlacionaram-se positivamente. 

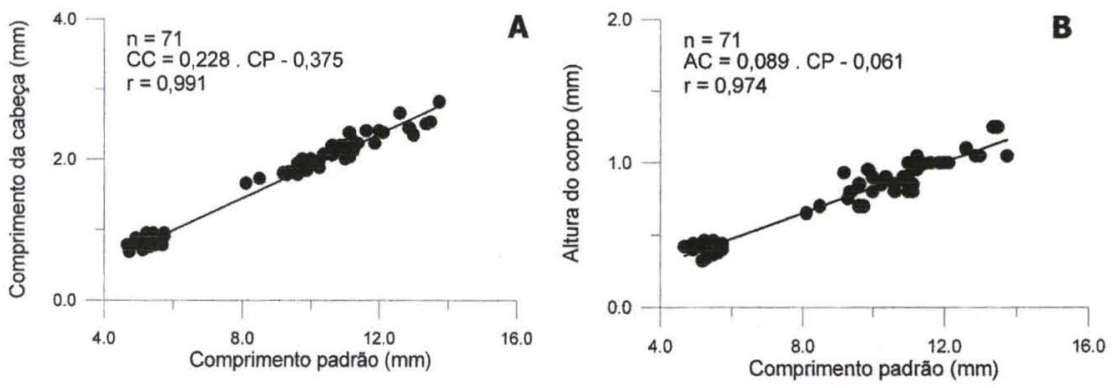

C
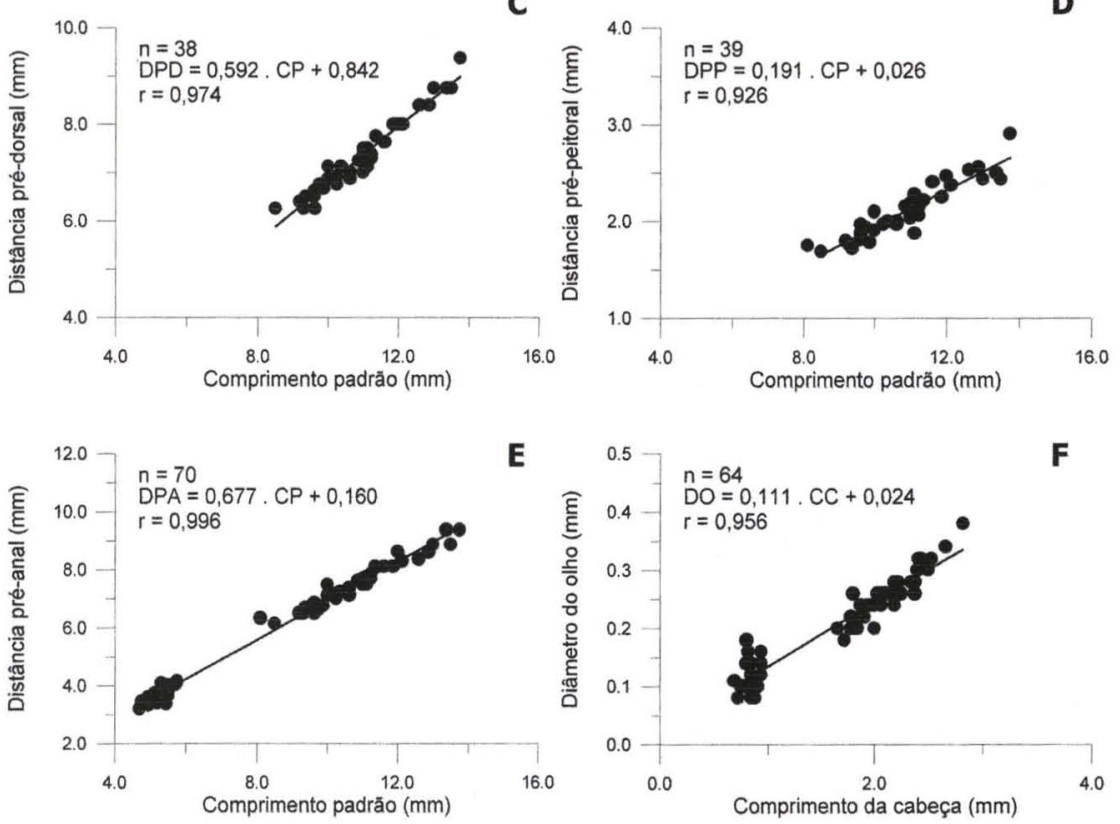

Fig. 2. Regressão linear de: (A) comprimento da cabeça (CC); (B) altura do corpo (AC); (C) distância pré-dorsal (DPD); (D) distância pré-peitoral (DPP); (E) distância pré-anal (DPA); (F) diâmetro do olho (DO) de Rhaphiodon vulpinus.

\section{Relações corporais}

As relações corporais das larvas de $R$. vulpinus, para as diferentes medidas e amplitudes de tamanho analisadas, são apresentadas na tabela I. Estas não apresentaram grande variação ao longo de seu desenvolvimento, conforme pode-se evidenciar na figura 3.

\section{DISCUSSÃO}

A forma e o tamanho do corpo nos diferentes estágios de vida, o desenvolvimento de espinhos e ornamentação dos ossos da cabeça e região cleitral, a presença de espinhos e raios moles nas nadadeiras, o seu desenvolvimento e padrão de 

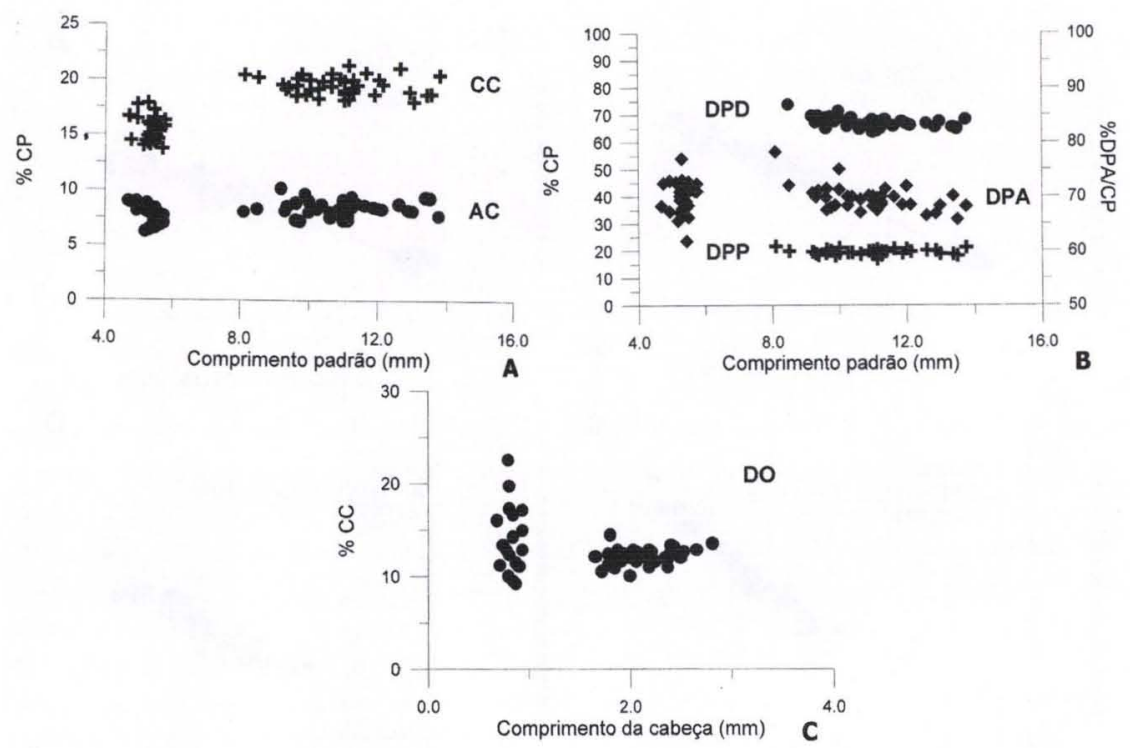

Fig. 3. Relações corporais de Rhaphiodon vulpinus: (A) comprimento da cabeça (CC) e altura do corpo (AC); (B) distâncias pré-nadadeiras (DPD) dorsal, (DPA) anal e (DPP) peitoral; (C) diâmetro do olho (DO).

Tabela I. Relações corporais de larvas de Rhaphiodon vulpinus.

\begin{tabular}{lccc}
\hline \multirow{2}{*}{ Medidas } & \multicolumn{2}{c}{ Relações corporais } \\
\cline { 2 - 4 } & Amplitude $(\mathrm{mm})$ & Porcentagem & Medida de referência \\
\hline Distância pré-dorsal & $8,5-13,8$ & $63,6-73,5$ & $\mathrm{CP}$ \\
Distância pré-anal & $4,7-5,8$ & $61,8-77,0$ & $\mathrm{CP}$ \\
& $8,1-13,8$ & $65,7-78,2$ & $\mathrm{CP}$ \\
Distância pré-peitoral & $8,1-13,8$ & $16,8-21,6$ & $\mathrm{CP}$ \\
Altura do corpo & $4,7-5,8$ & $6,2-9,0$ & $\mathrm{CP}$ \\
& $8,1-13,8$ & $7,2-10,1$ & $\mathrm{CP}$ \\
Comprimento da cabeça & $4,7-5,8$ & $13,7-17,3$ & $\mathrm{CP}$ \\
& $8,1-13,8$ & $18,0-21,3$ & $\mathrm{CC}$ \\
Diâmetro do olho & $0,7-0,9$ & $9,1-22,5$ & $\mathrm{CC}$ \\
\hline
\end{tabular}

pigmentação do corpo, assim como o número de miômeros representam caracteres comumente empregados para a identificação e diferenciação taxonômica de larvas de peixes. O padrão de escamação pode ser relevante, notadamente para espécies com escamas precoces (KENDALL et al. 1984).

A despeito das diferenças entre o desenvolvimento dos elementos das nadadeiras anal e dorsal de $R$. vulpinus, pode-se dizer que estas desenvolvem-se de forma praticamente simultânea, apesar de não terem sido avaliados exemplares com estruturas completamente formadas para nenhuma nadadeira, na faixa de tamanho analisado. Esta hierarquia na seqüência de formação das nadadeiras de $R$. vulpinus é similar à de outros Characiformes, como Mylossoma paraguayensis Norman, 
1929 (OldANI 1983a), Apareiodon affinis (Steindachner, 1879) (OLDANI 1977; BIALETZKI et al. 1998) e Semaprochilodus insignis Jardine \& Schomburgk, 1841 (ARAÚJO-LIMA 1985), não sendo útil portanto, para a diferenciação entre larvas de espécies desta ordem já estudadas.

NAKATANI et al. (no prelo) analisaram 50 larvas de $R$. vulpinus, abrangendo uma amplitude de 6,8 a 27,2 mm CP. Não foram constatadas diferenças entre as relações corporais apresentadas por aqueles autores e no presente trabalho, exceto para o diâmetro do olho. Esta última relação sofreu maior variação em larvas com CP entre 4,7 e 5,8 mm (Fig. 3A, F), faixa de tamanho não contemplada por aqueles autores, cuja ausência de pigmentação nos olhos, torna difícil a sua medição.

Dentre as larvas de Characiformes, cujas relações corporais são apresentadas na tabela II, evidencia-se que $R$. vulpinus e $A$. affinis apresentam menores proporções e amplitudes de variação de altura do corpo, enquanto que $R$. vulpinus caracteriza-se por possuir menor diâmetro do olho. Apesar de, no presente trabalho, só terem sido analisados indivíduos até a fase de flexão, e NAKATANI et al. (no prelo) só terem incluido dois exemplares na fase de pós-flexão em sua análise, os resultados sugerem que estas relações não sofrem grandes modificações ao longo do desenvolvimento larval.

Tabela II. Variação das relações corporais de larvas de Characiformes. (CC) Comprimento da cabeça, (CP) comprimento padrão, (DO) diâmetro do olho.

\begin{tabular}{|c|c|c|c|c|}
\hline \multirow{2}{*}{ Espécies } & \multicolumn{4}{|c|}{ Relações corporais } \\
\hline & $\mathrm{CC}(\% \mathrm{CP})$ & $\mathrm{AC}(\% \mathrm{CP})$ & DO (\%) & \\
\hline Apareiodon affinis (Steindachner, 1879) (1) & $9,3-38,0$ & $8,9-13,6$ & $28,3-34,5$ & (CC) \\
\hline Mylossoma aureum (Agassiz, 1829) (2) & $15,0-36,0$ & $11,0-57,0$ & $5,0-14,0$ & (CP) \\
\hline Mylossoma duriventre (Cuvier, 1818) (2) & $13,0-37,0$ & $8,0-55,0$ & $4,5-15,0$ & (CP) \\
\hline Mylossoma paraguayensis Norman, 1929 (3) & $23,0-34,0$ & $31,0-66,0$ & - & \\
\hline Piabucina pleurotaenia Regan, 1903 (4) & $15,4-27,6$ & $15,1-28,6$ & $35,0-50,0$ & (CC) \\
\hline Potamorhina altamazonica (Cope, 1878) (5) & $6,0-27,0$ & - & $2,0-7,0$ & (CP) \\
\hline Prochilodus scrofa (= P. lineatus) (Val., 1836) (6) & $17,8-38,4$ & $14,2-35,8$ & $20,0-29,0$ & (CC) \\
\hline Psectrogaster amazonica Eigen. \& Eigen., 1889 (5) & $7,0-38,0$ & - & $2,0-10,0$ & $(\mathrm{CP})$ \\
\hline Semaprochilodus insignis Jard. \& Schomburgk, 1841 (7) & $10,0-38,0$ & $15,0-45,0$ & $20,0-33,0$ & $(\mathrm{CC})$ \\
\hline Thoracocharax stellatus (Kner, 1858) (8) & $17,0-27,0$ & $11,0-43,0$ & - & \\
\hline Triportheus paranensis (Günther, 1874) (9) & $21,0-27,0$ & $13,0-28,0$ & - & \\
\hline Rhaphiodon vulpinus Agassiz, 1829 (10) & $15,6-22,4$ & $9,6-14,8$ & $9,6-17,6$ & $(\mathrm{CC})$ \\
\hline Rhaphiodon vulpinus (11) & $13,7-21,3$ & $6,2-10,1^{*}$ & $\begin{array}{r}9,14-22,5 \\
1,5-3,4\end{array}$ & $\begin{array}{l}\text { (CC) } \\
\text { (CP) }\end{array}$ \\
\hline
\end{tabular}

(1) BialetZKi et al. (1998), (2) Araújo-Lima et al. (1993), (3) Oldani (1983a), (4) Machado-AlliSon (1974), (5) Nascimento \& ARAújo-Lima (1993), (6) CAVICCHIOLI \& LeONHARdt (1993), (7) Araúso-Lima (1985), (8) Oldani (1979a), (9) Oldani (1979b), (10) NAKATANI et al. (no prelo), (11) presente trabalho, $\left(^{\star}\right)$ altura no início da nadadeira dorsal.

NAKATANI et al. (no prelo) constataram a presença de pigmentação nas larvas da espécie, inicialmente restrita para a região posterior do corpo e focinho, e, a partir da fase final de flexão, concentrando-se ao longo da linha lateral, região ventral da porção posterior do corpo e entre os raios da nadadeira caudal. No presente trabalho, entretanto, não foram evidenciados pigmentos nas larvas 
analisadas, as quais apresentaram uma coloração bege clara ou pardacenta, conforme sua coleta em áreas lacustres ou na calha do rio. Esta variação cromática pode estar relacionada às diferenças nos níveis de turbidez da água nas duas áreas, como resposta adaptativa à predação. Diferenças na coloração de exemplares adultos, preservados em álcool, provenientes de diferentes localidades, foram registradas por TOLEDO-PIZA (2000).

O número de vértebras, acrescido de uma unidade, apresenta uma correspondência com o número de miômeros (ARAúJo-LiMA \& DONALD 1988). O número de miômeros e vértebras têm grande importância na identificação de larvas de Cypriniformes (e. g. FUIMAN et al. 1983).

ARAúJo-Lima \& DonALD (1988) investigaram a utilidade destes dados merísticos como auxiliares na identificação das larvas de 29 espécies de Characiformes, comumente encontradas na calha central do rio Solimões/Amazonas. Estes autores encontraram 32 grupos de espécies organizados pelo número de miômeros estimados. Para a maioria das espécies de Characiformes do rio Amazonas, a utilidade do número de vértebras e, por conseguinte, do número de miômeros, para identificar suas larvas, ficou limitado pelas sobreposições encontradas. Porém, $R$. vulpinus e $R$. gibbus Agassiz, 1829 (= Cynodon gibbosus Agassiz, 1829 sensu TOLEDO-PIZA \& LAZARA 2000) foram enquadradas em grupos distintos, apresentando números de vértebras extremos, de 64 a 67 e 52 a 53, respectivamente, sem sobreposição com outras espécies. Para as duas espécies, o número de miômeros torna-se um fator de grande relevância para a identificação de suas larvas, já que são os únicos Characiformes estudados com número tão elevado de miômeros. No presente trabalho, foi encontrado um número máximo de 69 miômeros, duas unidades a mais que o número máximo de vértebras encontradas em $R$. vulpinus por ARAúJO-LIMA \& DONALD (1988). Esta diferença é aceitável, visto que estes autores também encontraram uma amplitude de variação ligeiramente superior na distribuição do número de miômeros em relação à distribuição de contagem de vértebras, atribuindo-a a erros experimentais (causados pela dificuldade em se visualizar o primeiro e último segmentos) e/ou a uma maior variabilidade real no número de miômeros. Ainda, deve-se levar em conta que as larvas estudadas neste trabalho pertencem a uma população da bacia hidrográfica do Paraguai, enquanto ARAÚJO-LIMA \& DONALD (1988) trabalharam com indivíduos da bacia amazônica. Por outro lado, um máximo de 69 miômeros também foi detectado por NAKATANI et al. (no prelo).

A presença de uma série externa de dentes (extra-orais) em larvas de Cynodontinae, considerada como sinapomorfia da subfamília por BUCKUP \& POIVRE (1994 apud TOLEDO-PIZA 2000), registrada em larvas de $R$. vulpinus a partir de $8,1 \mathrm{~mm} \mathrm{CP}$, representa um caractere útil na diferenciação deste taxa dentre os Characiformes. Em bacias onde os três gêneros são simpátricos, como a do Tocantins (SANTOS et al. 1984), a posição de inserção da nadadeira dorsal anterior à anal em Hydrolicus e coincidente ou ligeiramente posterior em Cynodon e Rhaphiodon - e o número de vértebras - 51-54 em Cynodon e 62-68 em Rhaphiodon (TOLEDO-PIZA 2000) -, podem ser úteis para a diferenciação de suas larvas. 
AGRADECIMENTOS. Ao Projeto Ecologia do Gran Pantanal (UFMT, IB/Max Planck Institut für Limnologie, Plön), integrante do Programa SHIFT (Studies of Human Impact on Forests and Floodplains in the Tropics, Env. 13), pelo apoio logístico e financeiro para a realização das coletas. Ao CNPq e CAPES, pela concessão de bolsa de doutorado a W. Severi (PPGERN/UFSCar) e à CAPES, pela concessão de bolsa PET a W. T. Z. Sousa (PET-Pesca/UFRPE). Aos comentários e sugestões apresentados por dois revisores anônimos.

\section{REFERÊNCIAS BIBLIOGRÁFICAS}

ABLE, K.A. \& M.P. FAHAy. 1998. The first year in the life of estuarine fishes in the Middle Atlantic Bight. New Brunswick, Rutgers Univ. Press, 342p.

Araújo-Lima, C.A.R.M. 1985. Aspectos biológicos de peixes Amazônicos. V. Desenvolvimento larval do Jaraqui-escama grossa, Semaprochilodus insignis (Characiformes, Pisces) da Amazônia Central. Rev. Brasil. Biol. 45 (4): 423-431.

ARAúJo-Lima, C.A.R.M. 1991. A larva da branquinha comum, Potamorhina latior (Curimatidae, Pisces) da Amazônia Central. Rev. Brasil. Biol. 51 (1): 45-56.

ARaújo-Lima, C.A.R.M. \& E. Donald. 1988. Número de vértebras de Characiformes do rio Amazonas e seu uso na identificação de larvas do grupo. Acta Amazonica 18 (1-2): 351-358.

Araújo-Lima, C.A.R.M.; A.L. Kirovsky \& A. MarCa. 1993. As larvas dos Pacus, Mylossoma spp (Teleostei; Characidae), da Amazônia Central. Rev. Brasil. Biol. 53 (4): 591-600.

Bialetzki, A.; P.V. SAnChes; G. Baumgartner \& K. NAKATANi. 1998. Caracterização morfológica e distribuição temporal de larvas e juvenis de Apareidon affinis (Steindachner) (Osteichthyes, Parodontidae) no alto rio Paraná, Paraná. Revta bras. Zool. 15 (4): 1037-1047.

BlaXTER, J.H.S. 1984. Ontogeny, systematics and fisheries, p. 1-6. In: H.G. Moser; W.J. RICHARds; D.M. Cohen; M.P. Fahay; A.W. Kendall JR. \& S.L. Richardson (Eds). Ontogeny and systematics of fishes. Lawrence, Amer. Soc. Ichthy. Herpet. Spec. Publ. 1, 640p.

Britski, H. A.; K.Z. Silimon \& B.S. Lopes. 1999. Peixes do Pantanal - Manual de Identificação. Brasília, EMBRAPA, 184p.

CAvicchioli, M. \& J.H. Leonhardt. 1993. Estudo do desenvolvimento morfológico de larvas de Curimbatá, Prochilodus scrofa (Steindachner, 1882), obtidas de reprodução induzida. Rev. UNIMAR, Maringá, 15 (Supl.): 109-124.

DiNGERKUS, G. \& L.D. UHLER. 1977. Enzyme clearing of alcian blue stained whole small vertebrates for demonstration of cartilage. Stain Technol. Baltimore, 52 (4): 229-232.

Fowler, H.W. 1950. Os peixes de água doce do Brasil. Arq. Zool., São Paulo, 6: 205-404.

Fuiman, L.A.; J.V. Conner; B.F. LATHrop; G.L. BuynaK; D.E. SNyder \& J.J. Loos. 1983. State of art of identification for cyprinid fish larvae from Eastern North America. Trans. Amer. Fish. Soc. 112: 319-332.

Godoy, M. P. 1986. Peixes e pesca do rio Paraná: área do futuro reservatório de Ilha Grande. Florianópolis, Eletrosul, 148p.

Goulding, M. 1980. The fishes and the forest - explorations in Amazonian natural history. Berkeley, Univ. California Press, 280p.

Kendall JR., A.W.; E.H. Ahlstrom; H.G. Moser. 1984. Early life history stages of fishes and their characters, p. 11-22. In: H.G. Moser; W.J. Richards; D.M. CoHEN; M.P. FAHAY; A.W. Kendall JR. \& S.L. RichARDSON (Eds). Ontogeny and systematics of fishes. Lawrence, Amer. Soc. Ichthy. Herpet. Spec. Publ. 1, 640p.

LASKER, R. 1987. Use of fish eggs and larvae in probing some major problems in fisheries and aquaculture. Amer. Fish. Soc. Symp. Bethesda, 2: 1-16.

Lesiuk, T. P. \& C.C. LindSEY. 1978. Morphological peculiarities in the neck-bending Amazonian characoid fish Rhaphiodon vulpinus. Can. Jour. Zool. 56: 991-997.

LuCENA, C.A.S. \& N.A. Menezes. 1998. A phylogenetic analysis of Roestes Günther and Gilbertolus Eigenmann, with a hypothesis on the relationships of the Cynodontidae and Acestrorhynchidae 
(Teleostei: Ostariophysi: Characiformes), p. 261-278. In: L.R. MALABARba; R.E. ReIS; R.P. VARI; Z.M.S. LuCENA \& C.A.S. LuCENA (Eds). Phylogeny and classification of Neotropical fishes. Porto Alegre, EDIPUCRS, 603p.

Machado-Allison, A. 1974. Etapas del desarrolo del pez Piabucina pleurotaenia Regan, 1903 (Characiformes: Lebiasinidae). Acta Biol. Venez. 8 (3-4): 579-622.

Moser, H.G.; W.J. Richards; D.M. Cohen; M.P. FahaY; A.W. Kendall JR. \& S.L. Richardson. 1984. Ontogeny and systematics of fishes. Lawrence, Amer. Soc. Ichthy. Herpet. Spec. Publ. 1, 640p.

Nakatani, K.; G. Baumgartner \& M.S. TenóRio-Baumgartner. 1997. Larval development of Plagioscion squamosissimus (Heckel) (Perciformes, Sciaenidae) of Itaipu reservoir (Paraná river, Brazil). Revta bras. Zool. 14 (1): 35-44.

NAKATANI, K.; G. BAUMGARTNER \& J.D. LATINI. 1998. Morphological description of larvae of the mapará Hypophthalmus edentatus (Spix) (Osteichthyes, Hypophthalmidae) in the Itaipu reservoir (Paraná river, Brazil). Revta bras. Zool. 15 (3): 687-696.

Nakatani, K.; A.A. Agostinho; G. Baumgartner; A. Bialetzki; P.V. Sanches \& M. Cavicchioli. (no prelo). Ovos e larvas de peixes de água doce: desenvolvimento e manual de identificação. Rio de Janeiro, Eletrobrás, 360p.

NAscimento, F.L. \& C.A.R.M. ARAúso-Lima. 1993. Descrição de larvas de Psectrogaster amazonica e Potamorhina altamazonica (Curimatidae, Pisces) da Amazônia Central. Acta Amazonica 23 (4): 457-472.

Oldani, N.O. 1977. Identificacion y morfologia de larvas de Apareiodon affinis (Steindachner) (Pisces, Parodontidae). Physis, Secc. B 37 (93): 133-140.

-1979a. Identificacion y morfologia de larvas y juveniles de Thoracocharax stellatus (Kner, 1860). (Pisces, Gasteropelecidae). Rev. Asoc. Cienc. Nat. Litoral 10: 49-60.

-1979b. Identificacion y morfologia de larvas y juveniles de Triportheus paranensis (Günther, 1874). (Pisces, Characidae). Rev. Asoc. Cienc. Nat. Litoral 10: 61-71.

- 1983a. Identificación y morfologia de larvas, juveniles y adultos de Mylossoma paraguayensis Norman, 1929. (Pisces, Characidae). Studies on Neotropical Fauna and Environment 18: 89-100.

-1983b. Identificacion y morfologia de las larvas y juveniles de Pimelodus maculatus Lacépède, 1803 (Pisces, Pimelodidae). Rev. Asoc. Cienc. Nat. Lit. 14 (2): 193-202.

SANTOS, G. M.; M. JEGU; B. DE MERONA. 1984. Catálogo de peixes comerciais do baixo rio Tocantins. Manaus, INPA, 83p.

Toledo-PizA, M. 2000. The Neotropical fish subfamily Cynodontinae (Teleostei: Ostariophysi: Characiformes): a phylogenetic study and a revision of Cynodon and Rhaphiodon. Amer. Mus. Novit. 3286: 1-88.

Toledo-Piza, M. \& K.J. Lazara. 2000. Cynodon Spix in Spix \& Agassiz, 1829 and Rhaphiodon Agassiz in Spix \& Agassiz, 1829 (Osteichthyes, Characiformes): proposed conservation, and proposed designation of $C$. gibbus and $R$. vulpinus Spix \& Agassiz, 1829 as the respective type species of Cynodon and Rhaphiodon. Bull. Zool. Nomencl. 57 (3): 151-157.

Recebido em 14.XII.2000; aceito em 28.I.2002. 